\title{
Short Telomeres in Patients with Vascular Dementia: An Indicator of Low Antioxidative Capacity and a Possible Risk Factor?
}

\author{
Thomas von Zglinicki, Violeta Serra, Mario Lorenz, Gabriele Saretzki, \\ Romana Lenzen-Großimlighaus, Reinhard Geßner, Angela Risch, and \\ Elisabeth Steinhagen-Thiessen
}

Institute of Pathology (TVZ, VS, ML, GS), Research Group Geriatrics (RL-G, ES-T) at the Evangelische Geriatriezentrum Berlin, and Institute of Laboratory Medicine and Pathobiochemistry (RG), Charité, Humboldt University, Berlin, and Department of Toxicology and Cancer Risk Factors (AR), German Cancer Research Center, Heidelberg, Germany

SUMMARY: Progressive cerebrovascular atherosclerosis and consecutive stroke are among the most common causes of dementia. However, specific risk factors for vascular dementia are still not known. Human telomeres shorten with each cell division in vitro and with donor age in vivo. In human fibroblasts in vitro, the telomere shortening rate decreased with increasing antioxidative capacity. There was a good intra-individual correlation between the age-corrected telomere lengths in fibroblasts and peripheral blood mononuclear cells. In 186 individuals including 149 geriatric patients (age range, 55-98 yr), leukocyte telomeres in patients with probable or possible vascular dementia were significantly shorter than in three age-matched control groups, namely in cognitively competent patients suffering from cerebrovascular or cardiovascular disease alone, in patients with probable Alzheimer's dementia, and in apparently healthy control subjects. No correlation was found to polymorphisms in the apolipoprotein E and glutathione-S-transferase genes. Telomere length may be an independent predictor for the risk of vascular dementia. (Lab Invest 2000, 80:1739-1747).

$P$ Posses rogressive cerebrovascular atherosclerosis and consecutively stroke are the second most common causes of dementia in Europe and the United States, and the most common causes in Asia and in many developing countries (Konno et al, 1997). The search for prognostic factors for late-onset dementia, and especially those with a vascular component, is a high research priority because (a) dementia is a major burden to any "greying" society, (b) stroke and vascular risk factors are not only causal for vascular dementia $(\mathrm{VaD})$ but also contribute to the pathogenesis of significant fractions of both Alzheimer's dementia (AD) and dementia with Lewy bodies cases, (c) dementia associated with stroke and vascular risk might be treatable or even preventable if patients at high risk could be identified early enough, (d) apart from age, there are no well-documented risk factors specifically for vascular dementia (Gorelick, 1997), and (e) the penetrance of identified or suspected genetic risk

Received July 13, 2000.

This work was supported by Verum Foundation, Munich, Germany, and by the Deutsche Forschungsgemeinschaft (Zg2/4-3).

Address reprint requests to: Dr. Thomas von Zglinicki, Department of Gerontology, IHE, Wolfson Research Centre, Newcastle General Hospital, Westgate Road, Newcastle upon Tyne NE4 6BE, UK. Fax: 44191219 5074;E-mail: t.vonzglinicki@ncl.ac.uk factors for late-onset dementia varies widely, limiting their use as prognostic factors.

Telomeres in somatic human cells shorten with each cell division in vitro, and telomere length in peripheral blood mononuclear cells (PBMC) (Frenck et al, 1998; Rufer et al, 1999) or endothelial cells (Chang and Harley, 1995) decreases with age. In immortal cells, including the vast majority of tumors, telomere shortening is counteracted by the activation of telomerase or, in few cases, of an alternative mechanism (Prescott and Blackburn, 1999). Ectopic expression of telomerase is sufficient to immortalize at least some human cells (Bodnar et al, 1998). Together, this data suggests a key role for telomeres as a biological clock, counting the successive rounds of replication and eventually triggering senescence in human cells. Maintenance of telomeres has been causally implicated in both hyperproliferative and hypoproliferative diseases (Chang and Harley, 1995; Prescott and Blackburn, 1999; Rufer et al, 1999). However, it has not been tested yet whether a connection between telomere maintenance in replicating cells and degenerative diseases of the same individuals might exist.

The causes of telomere shortening are only incompletely known. Both the inability of DNA polymerases to replicate a linear DNA molecule to its very end (Olovnikov, 1973) and the action of a strand-specific exonuclease (Makarov et al, 1997; Wellinger et al, 
1996) contribute to the shortening of telomeres. We have identified telomere-specific accumulation of DNA single-strand damage caused by oxygen free radicals as the major cause of telomere shortening in human fibroblasts in vitro by demonstrating that (a) telomeres in nonproliferating cells accumulate singlestrand breaks because of a specific deficiency in base excision repair (Petersen et al, 1998; von Zglinicki et al, 2000), (b) these breaks result in telomere shortening during DNA replication (Sitte et al, 1998), probably by transiently stalling the replication fork (von Zglinicki, 2000), (c) oxidative stress increases the telomere shortening rate by up to one order of magnitude (Saretzki et al, 1999; von Zglinicki et al, 1995, 2000), (d) diminution of oxidative stress slows down the rate of telomere shortening and decelerates replicative senescence (Sitte et al, 1998), (e) the telomere shortening rate correlates with the cell strain-specific antioxidative capacity (Serra et al, 2000). Some of these results were reproduced by others (Furumoto et al, 1998; Vaziri et al, 1997). In short, the relation of oxidative stress/antioxidative defense is the major determinant of the rate of telomere shortening in human fibroblasts in vitro. In other words, the agecorrected telomere length is a cumulative measure of the history of oxidative damage that a human fibroblast strain in vitro has experienced.

Cross-sectional studies indicate a gradual shortening of telomeres in PBMC in vivo with a constant rate at higher age (Frenck et al, 1998; Rufer et al, 1999). There are different factors influencing telomere kinetics in vivo, including genetic as well as chance factors that determine telomere length in the early embryo, telomerase activity, cell turnover rate, and factors that influence the telomere shortening rate over time. Few of these components have been unravelled. Telomerase activity in PBMC is low, especially at ages above 40 years (Iwama et al, 1998). Twin studies indicated that the PBMC telomere length is, to a large extent, genetically determined (Slagboom et al, 1994). Whether this is due to influences acting on the initial length or on the shortening rate is not known. However, a relationship between telomere length, oxidative damage, and antioxidative capacity seems to exist in vivo. Shorter telomeres were found in PBMC from patients with inherited respiratory chain disorders (Oexle and Zwirner, 1997) and from patients with Down syndrome (Vaziri et al, 1993), two conditions known to result in increased oxidative stress.

Taking into account the above mentioned in vitro data, it seems possible that the antioxidative capacity might be prominent among the factor(s) that control telomere length. If this is so, one would expect a good intra-individual correlation between age-corrected telomere lengths from different cell types, even if these had different replicative histories. Moreover, oxidative damage is thought to be of more serious consequence in nonproliferative cells and tissues. Therefore, one would expect a correlation of the age-corrected telomere length in any replicating cell type with the occurrence of degenerative heart and brain conditions that are known to be caused or accelerated by oxida- tive stress. Because of the cumulative nature of telomere loss, this correlation should become more obvious in old age.

Accordingly, the expectations mentioned above were tested first by correlating the telomere shortening rate to the antioxidative capacity of different primary human fibroblast strains. Secondly, the intraindividual correlation between the age-corrected telomere lengths in fibroblasts and PBMC was found to be strong, indicating that the age-corrected telomere length is, in fact, defined to a large extent by the genetic predisposition of the individual or by environmental or other physiological factors in the history of that individual rather than by the replicative history of a certain cell type. Finally, telomere length in PBMC from geriatric patients suffering from stroke, $\mathrm{VaD}$, or $A D$ was measured. Telomeres in patients with $\mathrm{VaD}$ were significantly shorter than in age-matched control subjects, in cognitively competent patients suffering from cerebrovascular or cardiovascular disease alone, or in patients with $A D$. There were no correlations found for either diagnosis or telomere length to polymorphisms in the ApoE and glutathione-S-transferase (GST) genes.

\section{Results}

\section{Telomere Shortening and Antioxidative Capacity in Fibroblasts In Vitro}

Telomere shortening rate ( $\Delta \mathrm{TRF})$ was measured over at least 20 population doublings in a number of different human fibroblast strains (Fig. 1A). Diploid foreskin (BJ) fibroblasts (with the longest replicative life span in vitro) maintain their telomeres best both under normoxia and hyperoxia. The skin fibroblast strains derived from study participants in our laboratory are intermediate in terms of telomere shortening rate, whereas the embryonic lung fibroblast strains WI-38 and MRC-5 display fast telomere shortening under control conditions and the highest shortening rate under hyperoxia. The latter two lines cease proliferation completely after 3 to 4 weeks under $40 \%$ hyperoxia (von Zglinicki et al, 1995, 2000).

Telomere shortening rates under normoxia (Fig. 1B) and hyperoxia (Fig. 1C) are correlated with the ability of these fibroblasts to compensate for an increased oxidative stress, ie, with their antioxidative capacity (AOC). Evidently, there are many possible confounding factors that contribute to the data variation, including not only experimental difficulties in measuring both $\triangle \mathrm{TRF}$ and AOC with high precision, but also the fact that the telomere shortening rate is a cumulative parameter, averaging the whole history of the strain, whereas the fluorescence measurement results in an acute estimate of the AOC. Still, the correlation between both parameters is statistically significant. This result is in accordance with previous data showing a dependency of the telomere shortening rate on hyperoxia-, hydrogen peroxide-, or alkylationmediated stresses (Saretzki et al, 1999; Vaziri et al 1997; von Zglinicki et al, 1995, 2000). It is further in 
A

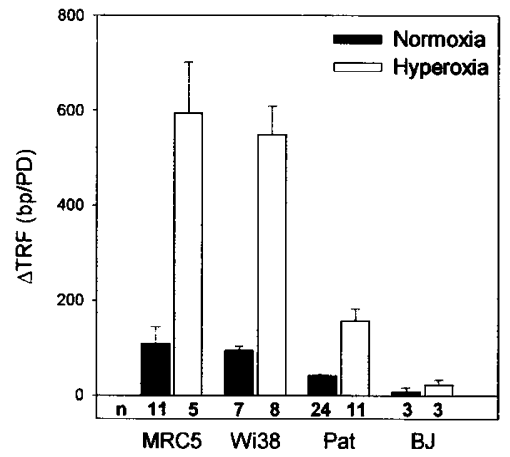

B

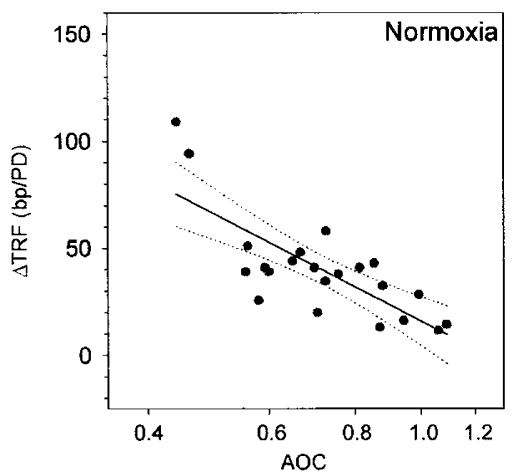

C

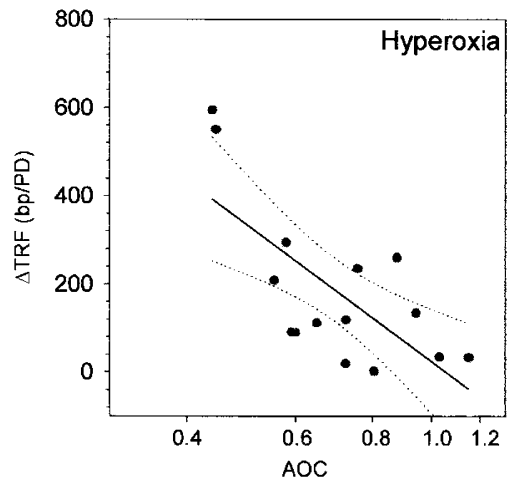

Figure 1.

Telomere shortening rate and antioxidative capacity in fibroblasts in vitro. $A$, Telomere shortening rate ( $\Delta$ TRF) is strain-dependent and increases under chronic hyperoxic stress. The numbers ( $\mathrm{n}$ ) of independent strains (patients [Pat]) or substrains (embryonic lung fibroblasts [MRC-5, WI-38] and diploid foreskin fibroblasts $[\mathrm{BJ}])$, respectively, are given. Data are mean \pm SEM. $B, \triangle \mathrm{TRF}$ vs antioxidative capacity $(\mathrm{AOC})$ under normoxic culture for the strains as given in $A$. Each patient fibroblast strain is given as an individual data point. Data are averages from between one and three independent experiments with standard errors of less than $20 \%$ of the estimates. The regression line and the $95 \%$ confidence interval are indicated. $C$, As in $B$, for the cells growing for 3 to 4 weeks under $40 \%$ normobaric hyperoxia.

accordance with observations indicating a correlation between telomere shortening rates in fibroblasts and the expression of the two major antioxidative enzymes, glutathione peroxidase and CuZn-superoxide dismutase (Serra et al, 2000). Together, this data indicates that the balance of oxidative stress and antioxidative protection is a major determinant of telomere length in telomerase-negative primary human fibroblasts in vitro.

\section{Intra-Individual Correlation between PBMC and Fibroblast Telomere Length}

Telomere length in PBMC is not only influenced by the replicative history and by oxidative damage/antioxidative defense. Granulocytes, naive and memory T lym- phocytes, and B lymphocytes do have different telomere lengths and their relative numbers change slightly with age (Rufer et al, 1999). The activity of telomerase, which is weakly present in different subtypes of PMBC in vitro (Iwama et al, 1998), might further complicate the age-dependency of the average PMBC telomere length. On the other hand, skin fibroblasts comprise comparatively homogeneous populations. They are devoid of telomerase activity. The replicative histories of PBMC and skin fibroblasts are surely different and probably unrelated.

Telomere lengths in PBMC and skin fibroblasts from 24 individuals out of all four study groups were compared (Fig. 2). The average telomere length in skin fibroblasts is about $2 \mathrm{~kb}$ longer than in PBMC, and the
A

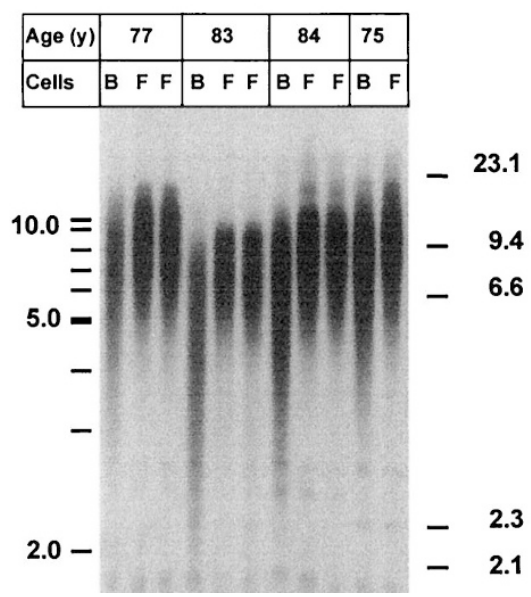

B

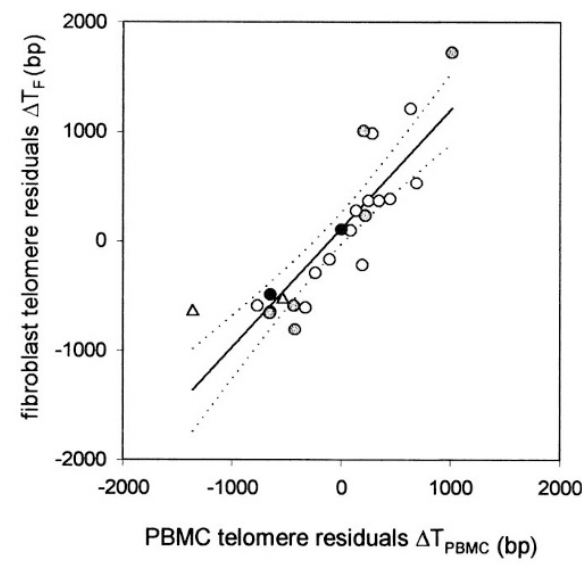

Figure 2.

Intra-individual correlation between telomere length in PBMC and skin fibroblasts. $A$, Telomere Southern blots from four individuals of similar age. Telomeres are either from PBMC (B) or from skin fibroblasts (F) at a population doubling level between 10 and 15 after explantation. For three patients, two parallel fibroblast experiments each are shown to indicate the amount of variation. Size markers (in $\mathrm{kbp}$ ) are indicated. $B$, Telomere length residuals (difference to average length) from 24 individuals. Data are from control individuals (open circles), patients with primary dementia (open triangles), patients with stroke and related risk factors (grey circles), and patients with probable vascular dementia (black circles). Data are averages of between three and seven independent measurements with standard errors of 50 to $350 \mathrm{bp}$. 
average telomere shortening rate with age is smaller (15bp/year in fibroblasts vs 20 bp/year in PBMC). However, a strong positive intra-individual correlation between PBMC and fibroblast telomere length was immediately evident in the Southern blot (Fig. 2A). To test whether this correlation was still valid after correction for in vitro telomere loss in fibroblasts and for age in vivo, we calculated first the telomere length in fibroblasts at explantation (ex vivo) from multiple measurements of both growth rate and telomere length in vitro. Second, we estimated the age-corrected telomere length residuals as the difference between the individual telomere length and the average value for that age as given by linear regression. Figure 2B indicates that a person with long telomeres in fibroblasts has always long telomeres in PBMC, and vice versa. The telomere length residual in an adult is specific for the individual, not for a cell or tissue type. This holds true even if the cells in question have a very different replicative history or if they differ with respect to the presence of active telomerase.

\section{Short Telomeres in PBMC from Patients with Vascular Dementia}

On both theoretical and experimental grounds, it has been suggested that the shortest telomeres should be related to cessation of cell division in primary cell lines. In fact, short telomeres were shown to accumulate in primary human fibroblasts before replicative senescence (Martens et al, 2000) and were maintained better than long ones in telomerase-transfected fibroblasts (Ouellette et al, 2000). Telomeric smears in PBMC are even broader than in fibroblasts (Fig. 2A). To find out whether the measurement of the length of the shortest telomeres might provide additional information, we measured the position of the shortest 10th percentile of PBMC telomeres, in addition to the average length in 40 patients, spanning the whole range of telomere lengths. We found a strong linear correlation between 10th percentile and average telomere length $\left(r^{2}=0.8939\right)$ with no indication for a deviation from linearity. This result indicates that there are no predominant effects acting on the shortest telomeres in our sample. Our finding is in accordance with data showing that the replicative capacity of fibroblast clones correlates with the average telomere length much better than with the length of the shortest telomeres (Martens et al, 2000).

Because the estimation of the average telomere length is much more robust experimentally, we measured this parameter rather than the length of the shortest telomeres in PBMC from 186 individuals in the age range between 18 and 98 years (Fig. 3). Between 3 and 6 independent measurements were performed per individual sample, resulting in a standard error of the estimates of less than $250 \mathrm{bp}$ in the vast majority of cases. The telomere length variation among different individuals of the same age is in the order of $2 \mathrm{kbp}$, in accordance with published results (Frenck et al, 1998; Rufer et al, 1999). Linear regression analysis reveals a significant decrease of the

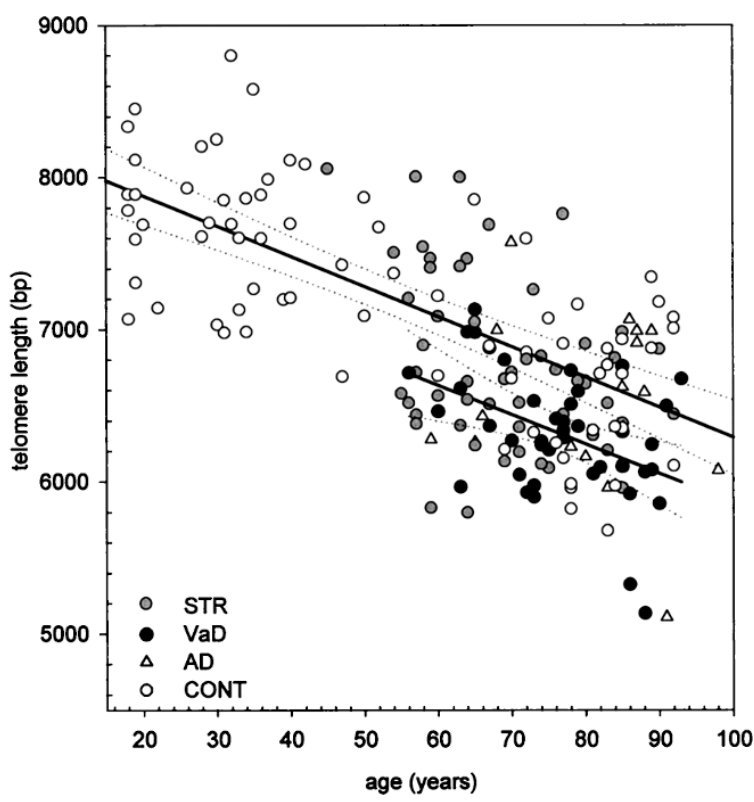

Figure 3.

Age dependency of telomere length in PBMC for control individuals (CONT, open circles), patients with probable Alzheimer's dementia (AD, open triangles), patients with stroke and related risk factors (STR, grey circles), and patients with probable or possible vascular dementia (VaD, black circles). Data are averages of between three and seven independent measurements with standard errors of 50 to $250 \mathrm{bp}$. The linear regression (straight lines) and 95\% confidence intervals (dotted lines) are given for the control and $\mathrm{VaD}$ group.

mean PBMC telomere length in the control group of 20 $\mathrm{bp} /$ year. Telomeres in PBMC from patients with $\mathrm{VaD}$ are significantly shorter than those from control subjects. The decline of the regression lines with age in both groups is about the same, but the average telomere length is about $440 \mathrm{bp}$ less in the $\mathrm{VaD}$ group. No patient with $\mathrm{VaD}$ had telomeres significantly longer than the average of those in the control group. To evaluate the statistical significance of these differences, we calculated the empirical frequency distributions for the PBMC telomere length residuals (differences between each telomere length and the agedependent average of the controls) for all four groups and compared those using Kolmogoroff-Smirnoff statistics (see the "Materials and Methods" section). Although the frequency distributions for controls and $A D$ patients were almost equal, a slight shift to shorter telomere residuals was found in stroke patients (including patients displaying severe cerebrovascular risk factors). However, this shift was not statistically significant $(p=0.28)$. On the other hand, the difference between the distributions for $\mathrm{VaD}$ patients and controls was highly significant $(p<0.001)$.

Telomere length-specific odds ratios were calculated as ratios of the disease-specific probability distribution to that of the controls (Fig. 4). For either stroke or primary dementia, the odds ratios did not deviate significantly from one, over the whole range of telomere residuals. On the contrary, the relative risk to suffer from vascular dementia was more than three times higher than normal for individuals with short 


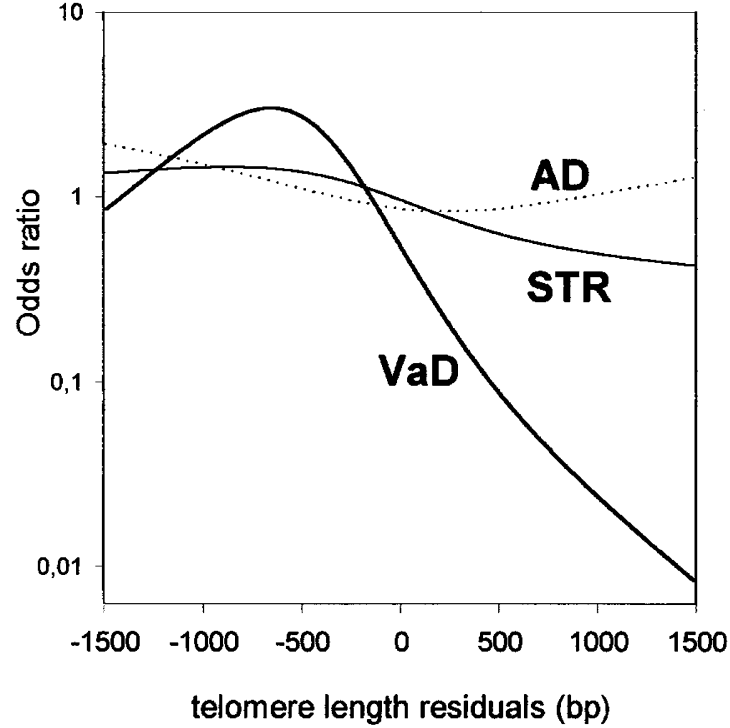

Figure 4.

Telomere length-specific odds ratios for stroke and related risk factors (STR), probable Alzheimer's dementia (AD), and probable or possible vascular dementia (VaD). Odds ratios for STR and AD are not significantly different from one over the whole range of telomere length residuals studied. However, the odds ratio for $\mathrm{VaD}$ decreases by up to two orders of magnitude in individuals with long telomeres and increases to more than 3 in patients with short telomeres.

telomeres, and it was up to two orders of magnitude lower than average for individuals with long telomeres.

\section{Other Risk Factors}

For $A D$ a number of risk factors is known, with the presence of the apolipoprotein $\mathrm{E}$ (ApoE) $\epsilon 4$ allele being the most important one for the spontaneous form of AD. Controversy exists regarding the association of the ApoE $\epsilon 4$ allele with $\mathrm{VaD}$ (Chapman et al, 1998; Marin et al, 1998), leading to the conclusion that ApoE is not a robust prognostic factor for this disease. In fact, despite its high incidence, no specific risk factors for $\mathrm{VaD}$ are known (Pohjasvaara et al, 1998). The distribution of ApoE polymorphisms was tested in a random subgroup of 75 patients (Table 1). One of these patients was homozygous for the ApoE $\epsilon 4$ allele; this patient was diagnosed clinically as AD. However,
21 out of these 75 patients were heterozygous for ApoE $\epsilon 4$, but there was no significant correlation of the presence of the ApoE $\epsilon 4$ allele with either diagnosis or telomere length.

Glutathione-S-transferases play an important role in the detoxification of xenobiotic substances, but also of intracellular aldehydes including the highly toxic 4-hydroxynonenal. Null polymorphisms exist in the GSTM1 and GSTT1 genes, and a A-G polymorphism at codon 104 of the GSTP1 gene has been found to reduce the activity of the protein (JourenkovaMironova et al, 1998). Decreased enzyme activities and/or the presence of these polymorphisms have been implicated in increased susceptibility to lung cancer, and in addition, as a possible risk factor for dementia (Lovell et al, 1998; Stroombergen and Waring, 1999). From the same subgroup as above, 11 patients were null for GSTT1, 37 were null for GSTM1, and 41 were hetero- or homozygously mutated at codon 104 of GSTP1. However, no significant correlation to either diagnosis or telomere length was found (Table 1). This holds for each of the GST genes alone as well as for the combined-risk genotypes. On the other hand, the telomere-length dependence of the incidence of $\mathrm{VaD}$ was clearly revealed in this group ( $p$ $=0.013$, Table 1). Together, these data demonstrate that telomere length correlated much more closely to $\mathrm{VaD}$ than established or suspected risk factors.

\section{Discussion}

The data presented here confirm oxidative stress as a major determinant of telomere length in human fibroblasts in vitro (Saretzki et al, 1999; Vaziri et al, 1997; von Zglinicki et al, 1995, 2000) and suggest that the antioxidative capacity of a cell strain might significantly modify the influence of externally or internally generated ROS on the telomere shortening rate. How oxidative stress/antioxidative defense determines telomere shortening is not completely clear. However, we have shown previously that it includes the accumulation of single-strand breaks in telomeres caused by telomere-specific low base excision repair efficiency (Petersen et al, 1998; von Zglinicki et al, 2000) and the transfer of these single-strand breaks into

Table 1. Correlation of ApoE and GST Genotypes with Vascular Dementia ${ }^{a}$

\begin{tabular}{|c|c|c|c|c|c|c|c|c|c|c|c|}
\hline & \multicolumn{2}{|c|}{ APOE } & \multicolumn{2}{|c|}{ GSTT1 } & \multicolumn{2}{|c|}{ GSTM1 } & \multicolumn{2}{|c|}{ GSTP1 } & \multicolumn{3}{|c|}{ TELO } \\
\hline & $\varepsilon 2, \varepsilon 3$ & $\varepsilon 4$ & wt & null & wt & null & wt & mut & long & average & short \\
\hline CONT & 11 & 8 & 17 & 2 & 8 & 11 & 8 & 11 & 7 & 7 & 5 \\
\hline STR & 14 & 3 & 11 & 4 & 7 & 8 & 10 & 5 & 3 & 9 & 5 \\
\hline$A D$ & 9 & 4 & 13 & 0 & 6 & 7 & 2 & 11 & 3 & 4 & 6 \\
\hline $\mathrm{VaD}$ & 19 & 7 & 21 & 5 & 15 & 11 & 12 & 14 & 0 & 8 & 18 \\
\hline$p$ (Chisq) & \multicolumn{2}{|c|}{0.440} & \multicolumn{2}{|c|}{0.209} & \multicolumn{2}{|c|}{0.748} & \multicolumn{2}{|c|}{0.057} & \multicolumn{3}{|c|}{0.013} \\
\hline
\end{tabular}

APOE, apolipoprotein E; GST, glutathione-S-transferase; TELO, telomere length distribution; CONT, controls; STR, patients with stroke-related diseases; AD, Alzheimer's dementia; VaD, vascular dementia; Chisq, Chisquare test.

${ }^{a} \mathrm{ApoE}$ and GST genotypes do not correlate to vascular dementia. A random subgroup of 75 patients (73 for GST) was analyzed for polymorphisms in the ApoE, GSTT1, GSTM1, and GSTP1 genes. Diagnostic groups are abbreviated as in Figure 3. Numbers of patients per group are given, and the error probabilities according to a Chisquare test are indicated. For comparison, the telomere length distribution (TELO) in these 75 patients is included. 
shortened telomeres during DNA replication (Sitte et al, 1998; von Zglinicki 2000).

That telomere length varies similarly in fibroblasts and PBMC from the same individual indicates that the mechanisms that govern telomere length are not specific to fibroblasts. Rather, the highly coordinated deviations from the average telomere length within each individual suggest that telomere length in different cells and tissues is under strong individual-specific genetic and/or environmental influence. A strong genetic component in telomere length regulation was found in twin studies (Frenck et al, 1998; Slagboom et al, 1994). The responsible genes have not been identified yet. However, first data revealed a coordinated increase of the abundance of glutathione peroxidase (GPX-1) and copper-zinc superoxide dismutase (CuZnSOD) mRNA in human fibroblast strains with low telomere shortening rate (Serra et al, 2000), implying two major antioxidative enzymes in telomere length regulation.

Figure 3 demonstrates a correlation between PBMC telomere length and the incidence of $\mathrm{VaD}$. Preferential telomere shortening could be a consequence of $\mathrm{VaD}$. However, this possibility is not very likely for these reasons: (a) According to evidence from crosssectional studies, telomeres in PBMC shorten monotonously but slowly with age. Except at very young childhood, a rate of $40 \mathrm{bp} / y e a r$ is hardly ever reached. A more than tenfold increase of the telomere shortening rate in patients with $\mathrm{VaD}$ would be necessary to explain the data given in Figure 3 as a consequence of the disease. (b) Our data show no significant correlation between telomere length and stroke or related risk factors. That means, short telomeres are not a consequence of stroke. It is hard to imagine, how the combination of stroke and dementia should contribute to telomere shortening, if neither stroke nor dementia does it alone. (c) The good correlation between PBMC and fibroblast telomere length (Fig. 2) rules out any factors that influence cell turnover or oxidative damage only in the blood stream as possible causes for the short telomere length in patients with vascular dementia.

Our data argues for individual-specific, probably genetic factor(s) as the major cause of the individual length of the telomere, in accordance with results from twin studies (Frenck et al, 1998; Slagboom et al, 1994). Those influences could either act on the telomere length at birth, on the telomere-shortening rate during adult life, or both. Because no longitudinal data are available, one cannot decide between these possibilities. However, we would like to suggest the individual's antioxidative capacity as an important factor in governing telomere shortening rate and adult telomere length. Individuals with less protection against ROSmediated damage would show a higher-than-average telomere-shortening rate. In case of stroke or severe cerebrovascular disease, their nerve cells would be less well protected against damage by free radicals, leading to a higher susceptibility to dementia. Oxidative stress resulting from the generation of both nitric oxide and superoxide is a major causal factor in ischemic and hemorrhagic stroke (Love, 1999). A major role of the antioxidant status of the brain in the occurrence and/or severity of $\mathrm{VaD}$ is well conceivable. This hypothesis is consistent with data documenting increased oxidative stress in patients with vascular dementia (Foy et al, 1999; Ihara et al, 1997; Tohgi et al, 1994).

Presently it remains still a possibility that the correlation between incidence of $\mathrm{VaD}$ and short telomere length as described here does not primarily reflect the low antioxidative capacity of the individual as a common cause. There might be other common underlying causes for both $\mathrm{VaD}$ and short PBMC telomere length. Assuming that telomere shortening is not a secondary consequence of vascular dementia, however, the conclusion would be in each case that short telomere length might be regarded as a risk factor for $\mathrm{VaD}$. Risks differ by more than two orders of magnitude (Fig. 4). The fact that telomeres, on average, shorten very slowly with age means that prospective risk assessment could be possible far in advance. Retrospective, as well as prospective, studies will be necessary to test this suggestion.

\section{Materials and Methods}

\section{Study Population}

The study population comprised of 37 clinically healthy volunteers and 149 patients admitted to the Evangelische Geriatriezentrum Berlin (EGZB) (81 males, 105 females, 18-98 years of age). The study population was not intended to resemble the average distribution of geriatric patients but was strongly selected for in favor of stroke and $\mathrm{VaD}$ patients. Cognitive abilities were assessed in all patients using the Minimal Mental State Examination (MMSE). Clinical diagnosis of mild-to-severe dementia was performed in 61 patients with a MMSE score of 24 and below, with additional confirmation by structured interview (SIDAM). In a number of these patients, further confirmation was obtained by either CT scan and/or MRI. Possible or probable dementia associated with cerebrovascular disease, as defined by the National Institute of Neurological Disorders and Stroke-Association Internationale pour la Recherche et l'Enseignement en Neurosciences (NINDS-AIREN) criteria (Roman et al, 1993), was diagnosed in 41 patients. Sixteen cases were diagnosed as Alzheimer's disease based on the diagnostic criteria of the DSM-IV (American Psychiatric Association, 1994) and the National Institute of Neurological and Communicative Disorders and Stroke-Alzheimer's Disease and Related Disorders Association (NINCDS-ADRDA) criteria for probable Alzheimer's disease (McKhann et al, 1984). Forty-four cognitively normal patients had a history of stroke, and 12 additional nondemented patients displayed severe cerebrovascular risk factors. Myocardial infarction, severe peripheral arterial occlusion, coronary heart disease in combination with either diabetes mellitus or hypercholesterinemia, or diabetes mellitus plus hypercholesterinemia were regarded as severe vascular risk 
factors in the present study. This 56 patients will be collectively referred to as "stroke group." The healthy control group consisted of 37 healthy volunteers (18-60 yr) and of 36 patients (52-92 yr) who were admitted to the EGZB for bone fractures or underwent hip surgery. These patients were without stroke or severe cardiovascular risk factors and scored 25 or higher in the MMSE test.

A $5 \mathrm{ml}$ heparinized blood sample was taken from all individuals. Samples were coded numerically and DNA extraction was started within 3 hours. The code was broken only after all telomere measurements were performed.

From 24 individuals (ages, 28-91 yr), a skin biopsy from the unexposed part of the left upper arm was taken after informed consent was obtained. This group included four patients with mild dementia. Two of these were probable $\mathrm{VaD}$. Furthermore, this group included six cognitively competent patients with stroke or stroke-related severe risk factors. The study was approved by the Humboldt University (Charité) Ethical Committee.

\section{Sample Preparation}

PBMC were obtained after density centrifugation of the diluted blood samples through Ficoll-Hypaque (Amersham Pharmacia Biotech, Uppsala, Sweden). Fibroblast cultures were obtained from the skin biopsies and grown in DMEM (BioWhittaker, Walkersville, Maryland) supplemented with $10 \%$ fetal calf serum, $1 \%$ glutamate, and $1 \%$ penicillin/streptomycin. WI-38 and MRC-5 primary lung fibroblast cultures were obtained from the American Type Culture Collection (ATCC, Manassas, Virginia), and primary BJ foreskin fibroblasts were obtained from Geron Corporation (Menlo Park, California). Cultures were grown for at least 20 population doublings (PD), and the growth rate was measured weekly at each passage. Growth rates were linear over the whole range in each case, and were used to extrapolate the cell number at $\mathrm{t}=0$ (explantation from the biopsy) and to calculate the population doubling level (PDL) from explantation in absolute terms. Telomere length was measured at three to four different time points, and these data were used to extrapolate the fibroblast telomere length at explantation.

\section{Telomere Length}

For preparation of highest quality genomic DNA, cells were embedded in $0.65 \%$ low-melting agarose plugs at a density of $10^{7}$ cells $/ \mathrm{ml}$ before deproteination by proteinase K treatment (Petersen et al, 1998; Sitte et al, 1998). DNA was digested until completion with Hinfl (60 units per plug; Boehringer Mannheim, Mannheim, Germany) at $37^{\circ} \mathrm{C}$. Plugs were analyzed in a $1.0 \%$ agarose gel by pulsed field gel electrophoresis (BioRad, Hercules, California). Gels were blotted to Hybond $\mathrm{N}+-$ membranes and hybridized with the telomeric probe (TTAGGG) ${ }_{4}$, conjugated directly to alkaline phosphatase (Promega, Madison, Wisconsin). A chemiluminescence signal was recorded on film within the linear range and analyzed in an imaging densitometer (BioRad). The average telomere length was calculated as the weighted mean of the optical density as described (Petersen et al, 1998). To standardize measurements between different blots, two different markers ( $1 \mathrm{~kb}$ ladder, and $\lambda$ HindIII) were used. In addition, at least five lanes per blot were used for repetition of measurements from previous blots.

\section{Antioxidative Capacity}

Fibroblast cultures were grown for at least three weeks in parallel under either normoxia (control) or $40 \%$ normobaric hyperoxia using a 3-gas cell culture incubator (Zapf Instruments, Sarstedt, Germany). Cells were stained with dihydrochlorofluoresceindiacetate, which became trapped and was oxidized by intracellular peroxides into the fluorescent dye, dihydrochlorofluorescin (DCF). The DCF fluorescence in $10^{4}$ cells was measured in a flow cytometer (PARTEC, Munster, Germany) using blue excitation and green emission wavelengths. The steady-state AOC was defined as the ratio DCF(normoxia)/DCF(hyperoxia), resulting in an $A O C$ of one for cell strains that can completely counteract the rise in intracellular peroxides under chronic hyperoxic stress.

\section{Genotyping}

Genotyping was performed in a random subgroup of 75 individuals, consisting of 17 stroke/risk factor patients (15 for GST genotyping), 26 patients with probable $\mathrm{VaD}, 13$ patients with $\mathrm{AD}$, and 19 control subjects. About $2 \mu \mathrm{g}$ DNA (one quarter of a plug each) was dissolved in $100 \mu \mathrm{H}_{2} \mathrm{O}$ and split to determine the following genotypes: GSTM1 (null vs positive), GSTT1 (null vs positive), GSTP1 (A-G polymorphisms at codon 104), ApoE ( $\epsilon 2, \epsilon 3, \epsilon 4)$. PCR and restriction analysis conditions were used as described earlier for GST (Jourenkova-Mironova et al, 1998) and ApoE analysis (Gessner et al, 1997).

\section{Statistics}

Telomere length was corrected for the influence of age using a Karhunen-Loewe transformation, ie, by calculating the residuals $\Delta T$ as the difference of each value to the mean length at that age as estimated by linear regression. Empirical frequency distributions $f(\Delta T)$ were calculated for the controls (C) and for each disease (D), and the distributions were tested for significant differences by using the Kolmogoroff-Smirnoff-test.

The empirical distributions were approximated by the sigmoidal regression curves $F(\Delta T)$ calculated by a least-squares approach. The quality of the fits, as estimated by Spearman's correlation coefficient $r^{2}$, was better than 0.99 in each case. The functions were differentiated to obtain the density distributions $\mathrm{dF}(\Delta \mathrm{T})$. The telomere length-specific odds ratio $\mathrm{OR}_{\mathrm{D}}(\Delta \mathrm{T})$ was calculated as the ratio $\mathrm{dF}_{\mathrm{D}}(\Delta \mathrm{T}) / \mathrm{dF}_{\mathrm{C}}(\Delta \mathrm{T})$.

To test the correlation between the presence of certain alleles and telomere length, PBMC telomere 
length was categorized as either short, average, or long. All estimates with standard errors that overlap with the 95\% confidence interval around the control regression line (Fig. 3) were regarded as average. Correlations were tested by using a Chisquare test.

\section{Acknowledgement}

The authors thank Dr. Peter Hufnagl (Berlin) for statistical advice, and Mrs. Susanne Metzkow (Berlin) and Mrs. Birgit Jäger (Heidelberg) for excellent technical assistance.

\section{References}

American Psychiatric Association (1994). Diagnostic and Statistical Manual of Mental Disorders, 4th ed. Washington: American Psychiatric Association Press.

Bodnar AG, Ouellette M, Frolkis M, Holt SE, Chiu CP, Morin GB, Harley CB, Shay JW, Lichtsteiner S, and Wright WE (1998). Extension of life-span by introduction of telomerase into normal human cells. Science 279:349-352.

Chang E and Harley CB (1995). Telomere length and replicative aging in human vascular tissues. Proc Natl Acad Sci USA 92:11190-11194.

Chapman J, Wang N, Treves TA, Korczyn AD, and Bornstein NM (1998). ACE, MTHFR, factor V Leiden, and ApoE polymorphisms in patients with vascular and Alzheimer's dementia. Stroke 29:1401-1404.

Foy CJ, Passmore AP, Vahidassr MD, Young IS, and Lawson JT (1999). Plasma chain-breaking antioxidants in Alzheimer's disease, vascular dementia and Parkinsons disease. QJM 92:39-45.

Frenck RW, Blackburn EH, and Shannon KM (1998). The rate of telomere sequence loss in human leukocytes varies with age. Proc Natl Acad Sci USA 95:5607-5610.

Furumoto K, Inoue E, Nagao N, Hiyama E, and Miwa N (1998). Age-dependent telomere shortening is slowed down by enrichment of intracellular vitamin $\mathrm{C}$ via suppression of oxidative stress. Life Sci 63:935-948.

Gessner R, Reischies FM, Kage A, Geiselmann B, Borchelt M, Steinhagen-Thiessen E, and Kottgen E (1997). In an epidemiological sample the apolipoprotein E4 allele is associated to dementia and loss of memory function only in the very old. Neurosci Lett 222:29-32.

Gorelick PB (1997). Status of risk factors for dementia associated with stroke. Stroke 28:459-463.

Ihara Y, Hayabara T, Sasaki K, Fujisawa Y, Kawada R, Yamamoto T, Nakashima Y, Yoshimune S, Kawai M, Kibata M, and Kuroda S (1997). Free radicals and superoxide dismutase in blood of patients with Alzheimer's disease and vascular dementia. J Neurol Sci 153:76-81.

Iwama H, Ohyashiki K, Ohyashiki JH, Hayashi S, Yahata N, Ando K, Toyama K, Hoshika A, Takasaki M, Mori M, and Shay JW (1998). Telomeric length and telomerase activity vary with age in peripheral blood cells obtained from normal individuals. Hum Genet 102:397-402.

Jourenkova-Mironova N, Wikman H, Bouchardy C, Voho A, Dayer P, Benhamou S, and Hirvonen A (1998). Role of glutathione-S-transferase GSTM1, GSTM3, GSTP1 and GST11 genotypes in modulating susceptibility to smokingrelated lung cancer. Pharmacogenetics 8:495-502.
Konno S, Meyer JS, Terayama Y, Margishvili GM, and Mortel KF (1997). Classification, diagnosis and treatment of vascular dementia. Drugs Aging 11:361-373.

Love S (1999). Oxidative stress in brain ischemia. Brain Pathol 9:119-131.

Lovell MA, Xie C, and Markesbery WR (1998). Decreased glutathione transferase activity in brain and ventricular fluid in Alzheimer's disease. Neurology 51:1562-1566.

Makarov VL, Hirose Y, and Langmore JP (1997). Long G tails at both ends of human chromosomes suggest a $C$ strand degradation mechanism for telomere shortening. Cell 88: 657-666.

Marin DB, Breuer B, Marin ML, Silvermann J, Schmeidler J, Greenberg D, Flynn S, Mare M, Lantz M, Libow L, Neufeld R, Altstiel L, Davies KL, and Mohs RC (1998). The relationship between apolipoprotein $\mathrm{E}$, dementia, and vascular illness. Atherosclerosis 140:173-180.

Martens UM, Chavez EA, Poon SS, Schmoor C, and Lansdorp PM (2000). Accumulation of short telomeres in human fibroblasts prior to replicative senescence. Exp Cell Res 256:291-299.

McKhann G, Drachman D, Folstein M, Katzman R, Price D, and Stadlan EM (1984). Clinical diagnosis of Alzheimer's disease: Report of the NINCDS-ADRDA Work Group under the auspices of the Department of Health and Human Services Task Force on Alzheimer's Disease. Neurology 34:939944.

Oexle K and Zwirner A (1997). Advanced telomere shortening in respiratory chain disorders. Hum Mol Genet 6:905-908.

Olovnikov AM (1973). A theory of margitonomy. J Theor Biol 41:181-190.

Petersen S, Saretzki G, and von Zglinicki T (1998). Preferential accumulation of single-stranded regions in telomeres of human fibroblasts. Exp Cell Res 239:152-160.

Ouellette MM, Liao M, Herbert BS, Johnson M, Holt SE, Liss HS, Shay JW, and Wright WE (2000). Subsenescent telomere lengths in fibroblasts immortalized by limiting amounts of telomerase. J Biol Chem 275:10072-10076.

Pohjasvaara T, Erkinjuntti T, Ylikoski R, Hietanen M, Vataja R, and Kaste M (1998). Clinical determinants of poststroke dementia. Stroke 29:75-81.

Prescott JC and Blackburn EH (1999). Telomerase: Dr. Jekyll or Mr Hyde? Curr Opin Genet Dev 9:368-373.

Roman GC, Tatemichi TK, Erkinjuntti T, Cummings JL, Masdeu JC, Garcia JH, Amaducchi L, Orgogozo JM, Brun A, Hofman A, Moody DM, O'Brien MD, Yamaguchi T, Grafman J, Drayer BP, Bennet DA, Fisher M, Ogata J, Kokmen E, Bermejo F, Wolf PA, Gorelick PB, Brek KL, Pajeau AK, Bell MA, DeCarli C, Culebras A, Korczyn AD, Bogousslavsky J, Hartmann A, and Scheinberg P (1993). Vascular dementia: Diagnostic criteria for research studies. Report of the NINDSAIREN International Workshop. Neurology 43:250-260.

Rufer N, Brümmendorf TH, Koolvra S, Bischoff C, Christensen K, Wadsworth L, Schulzer M, and Landsdorp PM (1999). Telomere fluorescence measurements in granulocytes and T lymphocyte subsets point to a high turnover of hematopoetic stem cells and memory $T$ cells in early childhood. J Exp Med 190:157-167. 
Saretzki G, Sitte N, Merkel U, Wurm RE, and von Zglinicki T (1999). Telomere shortening triggers a p53-dependent cell cycle arrest via accumulation of G-rich single stranded DNA fragments. Oncogene 18:5148-5158.

Serra V, Grune T, Saretzki G, and von Zglinicki T (2000). Telomere length as a marker of oxidative stress in primary human fibroblast cultures. Ann NY Acad Sci 908:327-330.

Sitte N, Saretzki G, and von Zglinicki T (1998). Accelerated telomere shortening in fibroblasts after extended periods of confluency. Free Radic Biol Med 24:885-893.

Slagboom PE, Droog S, and Boomsma DI (1994). Genetic determination of telomere size in humans: A twin study of three age groups. Am J Hum Genet 55:876-882.

Stroombergen MC and Waring RH (1999). Determination of glutathione-S-transferase $\mathrm{mu}$ and theta polymorphisms in neurological disease. Hum Exp Toxicol 18:141-145.

Tohgi H, Abe T, Nakanishi M, Hamato F, Sasaki K, and Takahashi S (1994). Concentrations of alpha-tocopherol and its quinone derivative in cerebrospinal fluid from patients with vascular dementia of the Binswanger type and Alzheimer type dementia. Neurosci Lett 174:73-76.

Vaziri H, Schächter F, Uchida I, Wei L, Zhu X, Effros R, Cohen D, and Harley CB (1993). Loss of telomeric DNA during aging of normal and trisomy 21 human leukocytes. Am J Hum Genet 52:661-667.
Vaziri H, West MD, Allsopp RC, Davison TS, Wu YS, Arrowsmith CH, Poirier GG, and Benchimol S (1997). ATMdependent telomere loss in aging human diploid fibroblasts and DNA damage lead to the post-translational activation of p53 protein involving poly(ADP-ribose)polymerase. EMBO J 16:6018-6033.

von Zglinicki T (2000). The role of oxidative stress in telomere length regulation and replicative senescence. Ann NY Acad Sci 908:99-110.

von Zglinicki T, Saretzki G, Döcke W, and Lotze C (1995). Mild hyperoxia shortens telomeres and inhibits proliferation of fibroblasts: A model for senescence? Exp Cell Res 220: 186-193.

von Zglinicki T, Pilger R, and Sitte N (2000). Accumulation of single-strand breaks is the major cause of telomere shortening in human fibroblasts. Free Radic Biol Med 28:64-74.

Wellinger RJ, Ethier K, Labrecque P, and Zakian VA (1996). Evidence for a new step in telomere maintenance. Cell 85:423-433. 